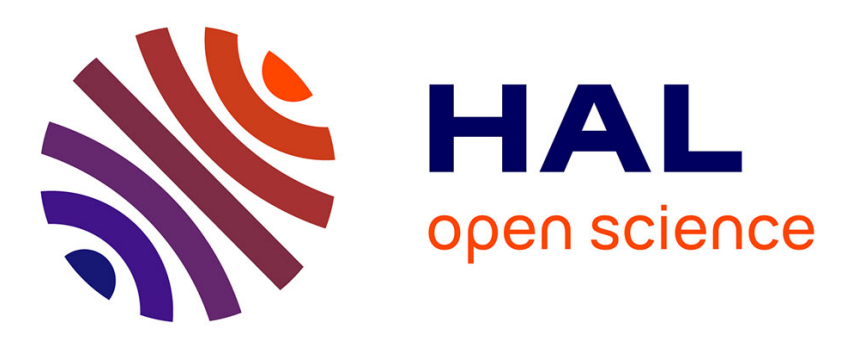

\title{
Mechanical self-stabilization, a working hypothesis for the study of the evolution of body proportions in terrestrial mammals
}

\author{
Rémi Hackert, Nadja Schilling, Martin S Fischer
}

\section{To cite this version:}

Rémi Hackert, Nadja Schilling, Martin S Fischer. Mechanical self-stabilization, a working hypothesis for the study of the evolution of body proportions in terrestrial mammals. Comptes Rendus. Palevol, 2006, 5 (3-4), pp.541-549. 10.1016/j.crpv.2005.10.010 . hal-00365982v1

HAL Id: hal-00365982

https://hal.science/hal-00365982v1

Submitted on 5 Mar 2009 (v1), last revised 10 Jul 2019 (v2)

HAL is a multi-disciplinary open access archive for the deposit and dissemination of scientific research documents, whether they are published or not. The documents may come from teaching and research institutions in France or abroad, or from public or private research centers.
L'archive ouverte pluridisciplinaire HAL, est destinée au dépôt et à la diffusion de documents scientifiques de niveau recherche, publiés ou non, émanant des établissements d'enseignement et de recherche français ou étrangers, des laboratoires publics ou privés. 


\title{
Mechanical self-stabilization, a working hypothesis for the study of the evolution of body proportions in terrestrial mammals?
}

\author{
Rémi Hackert a,b, Nadja Schilling ${ }^{a}$, Martin S. Fischer ${ }^{\text {a }}$
}

a Institut für Spezielle Zoologie und Evolutionsbiologie mit Phyletischem Museum. Erbertstr. 1 D-07743 Jena, Germany.

b FRE 2696 CNRS/MNHN/Paris6/Collège de France «Adaptations et Evolution des systèmes ostéomusculaires , Museum National Histoire Naturelle USM302/CP55/Pavillon d'Anatomie comparée, 57 rue Cuvier 75231 Paris cedex 05

\begin{abstract}
Locomotion is a behaviour resulting from the interaction of the nervous and muculo-skeletal systems and the environment. However the musculo skeletal systems of some terrestrial mammals present an intrinsic ability to realize a dynamic stable locomotion. Actual anthropomorphic passive walkers demonstrate that a pure mechanical system with leg and arms is able to walk down an inclined plane. Numerical simulations confirm that self-stabilization of the mechanics is acting in running too. The necessity to ensure the dynamic stability of a cyclic locomotion set physical constraints to the musculo-skeletal system. A description of the locomotor apparatus with neuromechanical variables like the stiffness - accessible to the experimentation- enables for maintaining the number of degrees of freedom of biomechanical models as low as possible. The maximization of the robustness of the mechanical self-stabilization of the models with regard to the body proportions represents for future simulations an optimization criterion which should bring a new light to the comprehension of the body proportions.
\end{abstract}

Keywords: stability locomotion body proportion running quadruped mammals. 


\section{Résumé}

La locomotion résulte de l'interaction du système nerveux, du système osteomusculaire et de l'environnement. Cependant le système musculo-squelettique de certains mammifères terrestres apparait avoir acquis une capacité intrinsèque à se mouvoir d'une façon dynamiquement stable. Les robot-marcheurs passifs actuels nous montrent qu'une structure mécanique avec des jambes et des bras est capable de descendre le long d'un plan incliné sans source de contrôle supplémentaire. Les simulations numériques semblent de plus montrer que des phénomènes d'autostabilisation de la mécanique sont à l'œuvre pendant la course également. La nécessité d'assurer une locomotion cyclique dynamiquement stable est porteuse, elle aussi, de contraintes physiques à définir, à découvrir. L'étude des relations entre stabilité dynamique et morphologie suppose une modélisation du système étudié et l'utilisation de l'outil simulation numérique. Une description du système avec des variables neuromécaniques telle la raideur, accessible à l'expérimentation, permet de contenir le nombre de degrés de liberté du modèle. La maximisation de la robustesse de l'autostabilisation mécanique, c'est-à-dire la maximisation de l'intensité de la perturbation que le système en mouvement est capable de supporter sans tomber, fournit pour les futures simulations un critère d'optimisation capable d'éclairer d'une façon nouvelle notre compréhension des proportions du squelette.

Mots-clé: stabilité dynamique, locomotion, proportion, segment, course, mammifères quadrupèdes. 


\section{Introduction}

By the transition from the sprawled reptilian leg configuration to the parasagittal mammalian limb, one additional segment was added in the fore and hind limbs (scapula and elongation of the tarsus). Thus, scapula and femur, humerus and tibia, ulna and tarsus became functional correspondent in mammals [44, 40]. During in-phase gaits (bound, halfbound, gallop), extensive flexions and extensions occur in the posterior thoracic and the lumbar regions of the spine especially in mammals [28]. These back movements act to some extent as an additional proximal segment modulating the position of the pelvis up to 40 degree [49]. Mammalian posture seems to follow the "crouched posture and high fulcrum" [20] i.e. scapular pivot in fore and hip joint in hind limbs are held at the same level. But intralimb proportions and relations between the functional corresponding segments within the kinematical chains are still poorly understood if well described by allometry relations within a family [42].

The locomotor apparatus evolved under the selective pressure of structural, (eco-) physiological and mechanical constraints. Among them efficiency in energy consumption is certainly one of the most important constraints and is expressed in two dual ways to handle with energy during (loco-) motion.

First: reduction of muscular work as much as possible. Kustnezov [40] interpreted the incorporation of the third segment as an energy saving adaptation which enables the reduction of the mechanical work of leg muscles against each other during the stance phase. The author calculates from his model the kinematics which minimizes muscular work during locomotion over flat ground and found a kinematics comparable to the kinematics observed in small mammals ( $[48,50]$ and overview in [24]).

Second: storage or conversion of kinetic energy during one phase to recover it in another phase of the movement [2, 3]. Cavagna et al. [12, 18] described two mechanisms: The inverted pendulum during slow motions and the elastic mechanism during fast movements. In the first case, kinetic and gravitational potential energy associated with the motion of the centre of body mass differ in their phase and this results in a transfer of kinetic into potential energy and back within the locomotor cycle. In contrast to this, kinetic and gravitational potential energies vary in phase in the elastic mechanism and thus there is no conversion between kinetic and gravitational potential energy during fast locomotion but in place the kinetic energy is provisory stored in elastic element - tendons and muscles [57, 41, 43] and is restored in a next phase of the movement. Elastic energy storage is assumed for small mammals as well as for larger mammals [12] although there is no need to assume it in order to explain their energy balance [37, 38]. 
The named strategies are a response to the physical constraints applied by interactions of the body with its environment. Mechanical interaction as one of the components of this interaction (beside physico-chemical) is often explained as the necessity to support the body weight i.e. resist gravitational forces. But this is also true during locomotion. Inverse dynamic analysis demonstrates that the net torques created in the joints by muscles work mainly against gravity $[60,22]$. However, anti gravitational action is only one vector of the mechanical interaction.

The stability of locomotion particularly at key events as escaping or hunting if stability and manoeuvrability are most important- is a second vector of this interaction. The study of the relationships between stability and morphology has to be embedded into one possible approach described by this contribution.

\section{Dynamic stability during locomotion}

A standing quadrupedal animal is in static equilibrium. The feet, as points of ground contact, map a polygon called polygon of support. As long as the vertical projection of its centre of body mass remains inside the polygon the animal is said to be statically stable. This is the case during slow walking which is described as a succession of quasi-static equilibrium. Three legs are always in contact to the ground. The triangle of support changes periodically and the vertical projection of the centre of mass then moves from one triangle to the next. At any time the animal is able to stop its forward motion if the speed is low and therefore the quantity of movement (mass time speed) is small. Cartmill et al. [10] show on the basis of a large set of footfall patterns that quadruped mammals prefer limb coordinations that maximize the time of existence of such a polygon of support during walking. If speed is increased, a preference for limb co ordinations that involve diagonal pairs of limbs is given. During trotting, the polygon is reduced to a diagonal line connecting one hand and the ipsilateral foot. Thus, trotting animals have already leaved the frame of a succession of quasi-static equilibrium state to meet dynamic equilibrium. Idem gallop, bound, half-bound.

Stability qualifies equilibrium. Equilibrium is said to be statically stable if a system returns to its initial equilibrium after a perturbation as for instance a pendulum after deflection from its vertical position. In case of a cyclic movement the motion of a system is said stable if it is able to go back to its nominal trajectory after perturbation. This restrictive definition of dynamic stability represents more than simply remaining on the feet. Since motion of a living organism underlies variability, the given definition is rarely realized in nature. In many studies on stability of motion systems, particularly in the robotics, a system is more pragmatically assumed stable if it reaches a neighbour stable state after a perturbation even if it does not return exactly to its nominal state. 


\section{Addressing the right degree of complexity}

Locomotion is generally described as the behaviour which results from the interaction of the musculo-skeletal system and the neural system and environment components. It is a complex task which exhaustive description would require hundreds of variables. The aim of modelization is to reduce the number of variables - i.e. the number of degrees of freedom (d.o.f.) - to a necessary minimum.

Due to the different physical mechanisms acting in slow and fast locomotion, the number of d.o.f. is reduced by addressing these two types of locomotion separately.

Applying Newton's first principle of dynamics, the forces exerted by the limbs on the ground (i.e. ground reaction forces) and measured by the use of ergo meters [11] can be integrated numerically two fold. The integration leads to the motion of the centre of mass (c.o.m.) of the body.

During trotting and in-phase gaits, motions of the centre of mass of mammals occur mainly in the parasagittal plane. At trot, gait symmetry and resulting torsions of the spine around the longitudinal body axis lead this parasagittal motion of the CoM. At in-phase gaits spine bending movements lead to extensive parasaggital movements of the pelvis which contribute up to half of the stride length in small mammals $[21,23,24,49]$. Therefore the lateral excursion of the centre of mass is smaller than its vertical ones. Thus, a modelization of a quadruped mammal during fast locomotion is reasonable in a two dimensional plane whereas a two dimensional model of slow locomotion will be a partial description of the reality. It is assumed that models deal with straight forward locomotion.

Most simulations studies of the last 15 years can be classified into three types which differ in the description of the interaction between neuronal and mechanical systems and emerged after the publication of three basic articles: Taga [55] modelized a rough musculoskeletal and a rough nervous system separately which interaction produces a stable cyclic motion. McGeer [31] studies the passive walking of a structure along an inclined plane, and investigates the passive properties of the mechanics disregarding voluntary the role of the nervous system. In direct line to the works of Cavagna and Taylor, Blickhan [7] proposed a synthetic model taking into account elastic energy storage during running: the spring-mass model. 


\section{Multibody dynamics}

Tagas' purpose [55] was to test whether a stable walk is able to emerge from the interaction of mechanical and nervous systems defined as follows: The mechanical structure was modelized by a series of rigid segments -three for each limb and a rigid trunk. Taga associates a couple of neuro-oscillators with each limb joint, an assumption founded on its existence in a primitive specie - the lamprey [30] and a presumption of its existence in other species. The localization of the neuro-oscillators was recently identified in a tetrapod [13]. Each pair of neuron influences the pair of the adjacent proximal joint. A relatively simple mathematical neuron model (integrator) was assumed. Torques acting at the joints were assumed proportional to the output signal of the neurons. Taga was able to get the model walking stably through the settings of the "right" values of the parameters into the model. This study became the core of others, integrating almost all bones and muscles [36], optical sense, and obstacle avoidance into the neuro-controller. With this, the number of degree of freedom of Tagas' basic model was considerably increased. The proposed neurocontrollers and their development largely founded the success of actual Japanese humanoid robots.

Morphologists can learn from complex simulations if they address the right level of complexity. But the quality of the extrapolations made with very complex models [e.g. 36] with hundred of variables is rather poor. Due to the extremely large parameters space and the redundancy in the models (however redundancy is also present in living systems), the search for optimal value of some choosen variable with regard to stability does not lead to significant results since changes in the value of one parameter can easily be compensated by the change of others.

Thus for the study of the evolution of musculo skeletal design it seems to be more promising to comprehend a construction step by step and reconstruct it stepwise. In the field opened by Taga's work - i.e. coupling of one mechanical and one neuronal system - as a morphologist the most interesting simulations are the attempt to discover how few features of the reality is necessary to let a given behaviour emerge. With this aim Ijspeert [56] investigates for instance the transition from a standing wave to a travelling wave during the locomotion of a salamander transiting from land to water.

\section{Passive walking dynamics}

McGeer [31] designed (originally) a toy that was able to walk down along a slope without additional external control, propelled only by the gravitation. Bipedal passive walker models and robots were then developed with anthropomorphic dimension with or without knees and/ or feets [26, 14, 32]. A periodic input of energy at the hip even 
enables a stable walk on a horizontal and even ground. More recently Collins et al. [15] developed a 3D simulation model includings arms which movements stabilized the model successfully [39]. Despite the fact that passive walker actually disregard some features of the walking motion (ankle extension at the end of the stance phase for instance), this main result demonstrates that the body mechanics do not only evolved in order to enable an economic walking but also a stable walking and that this mechanical stability is founded on synergies in the motion of the different part of the body. The results give raise to conceptual development. Following this, is it appropriate to subordinate the musculoskeletal system to the nervous system in the case of a cyclic (automatic) motion? Or should we accept the idea that mechanics control the neuronal system just like the neuronal system control mechanics [9]?

\section{Spring mass models for fast locomotion}

Blickhan [7] found a roughly linear relationship between the ground reaction force and the distance from the centre of mass to the point of ground contact at the preferred hopping frequency of humans. He introduced the spring mass system in order to model this relationship (figure 1A). The spring mass model has only five degrees of freedom (mass, spring stiffness, spring length, speed vector, angle of attack) and fitted the experimental data with a precision more than $20 \%$ in the range of measurement. It comes up to the requirement of Cavagna et al. [12] that the energy balance of middle and large sized mammals during fast locomotion cannot be explained without assuming elastic energy storage. The described linearity is surprising because force length relationships of an isolated muscle are not linear, the geometry of bones head articulation is not circular and thus the relation of the lever arm of a given muscle versus joint aperture is also not linear. The integration of these components leads first to a linear force-length relationship [59].

Farley et al. $[16,17]$ extended the validity of the spring mass model to the description of trotting quadrupeds. In this description the spring represents the action of a pair of diagonal limbs. Hackert et al. [33, 34] confronts the spring mass model to in-phase gaits of small mammals and showed that the force length relationships in forelimbs were also roughly linear in small mammals. But linearity is achieved as a consequence of the intense sagittal spine flexion at inphase gaits that shifts the centre of body mass cranially [35]. A roughly linearity of the force length relationships means that the stiffness - that means the ratio of the change in the force (measured in Newton) through the corresponding change in a length (deformation) - is almost constant during the stance phase of forelimbs. The role of limb's stiffness (in $\mathrm{Nm}^{-1}$ ) and thus the role of compliance - was already recognized as one determinant of locomotion [7][8][45][46] but its direct involvement in the achievement of a dynamic stable locomotion was demonstrated clearly only recently. 


\section{Stiffness of a structure and dynamic stability}

The stiffness of a musculo-skeletal structure can be split into two major components: (a) a passive component linked to the intrinsic properties of the materials and (b) an active component (depending on muscle activation and geometry). Muscles force does not necessary result in movement. If agonist and antagonist muscles are activated synchronously so that torques of agonist and antagonist muscles remain in equilibrium, no movement will occur in the articulation but its stiffness will be increased. Feldman [19] demonstrates that the control of only one parameter is sufficient to control joint stiffness and aperture.

Dynamic stability of the spring-mass system - i.e. its ability to bounce stably- was studied first by Schwind and Koditchek [51]. They derived an analytical criterion from the linearized (i.e. approximated) equation of motion. Seyfarth et al. [52] performed dynamic simulation of a non linearized model and demonstrate its ability to bounce stably for some combinations of the forward speed, the spring-leg stiffness and the angle of attack which is the angle spanned by spring-leg with the ground line at the instant of touch down. That is within a range of values of stiffness and angle of attack and mass position a stable behaviour can be generated (figure 1D). Thus small irregularities of the ground which would result in small changes of the mass position relative to the ground can be overcome without resetting the values of the system, i.e. without control. This concept of a mechanical stabilisation without external control is known in physics as self-stabilization.

Approximations are numerous by spring mass models. During forward movement the spring mass model describes only the stance phase of a spring-leg not the swing phase. The spring-leg is assumed to be massless and from the point of view of the dynamics a massless object cannot realize a motion. Thus, in the simulation the leg is artificially positioned with a given angle of attack during the flight phase of the spring mass system before the next ground contact. This is an important restriction but $85 \%$ of the mass is included in the head-trunk structure in small mammals. Limbs of small mammals are light indeed and therefore assuming massless legs is appropriate. Additionally the model assumes that ground reaction forces keep the direction of the spring -, towards the centre of mass and this is only true as well in humans as in quadruped at constant speed and during the middle stance phase [6].

The comparison between the spring mass system and galloping small mammals show additional descriptive limits. The simple spring mass model trajectory undulates with one maximum and one minimum per cycle whereas the centre of mass of galloping small mammals may have more than two extremum within each locomotor cycle. This gives raise to the development of extended springs-mass models in order to take the back dynamics into account (fig 1C). Hackert and Fischer [35] propose to see the flexion of the spine in small mammals and the associated forward displacement of 
the centre of mass as a way to adjust the angle of attack of fore limbs and thus to use mechanical self-stabilization mechanism. Back flexion co-determines the position of the centre of mass and back stiffness sets the rough instant of touch down of the forelimb by controlling the downwards motion of the centre of mass during hind limbs' stance phase. In respect to the simple spring mass system, Seyfarth et al. [54] implement a rotation of the spring leg during its late swing phase when the mass has reached the apex of its trajectory. With this, the authors show that a tuning of the instant and verticality of the leg at touch down may influence positively the dynamic stability of the system. This may explain the retraction of the fore limb observed in galloping mammals before touch down [24].

\section{Models and templates: Learning from neuromechanical models stepwise.}

The spring mass model developed from the status of a simple mechanical model for human hopping to a neuromechanical template. Full and Koditschek [25] proposed to face the spring mass template like an asymptotical limit to which running systems tend to. Moreover, the authors conceptualized a method (rather new in the field of motion studies) to handle with complex system involving a lot of degrees of freedom. First: reduce the number of degrees of freedom by projecting the problem onto one plane, second then model it in this plane, then progressively include new additional degrees of freedom. This method is comparable to the early stage of movement learning in child [5] when degrees of freedom are introduced and the stiffness of the limbs reduced stepwise during growth [47].

The introduction of the neuromechanical parameter "stiffness" leads to a level of description that enables provisory to "disregard" how the stiffness is generated i.e. to disregard the muscles, their insertion and activation. The number of degree of freedom of the biomechanical model becomes then considerably lower.

\section{Distribution of the global stiffness onto the joints}

The spring mass model is a model at the level of organisms. Limb stiffness can be compared between species after normalization by mass and length. Blickhan and Full [8] introduce the relative stiffness $k=\left(F_{\max } / m g\right) /(\Delta l / l)$ and found a values around 8 for animals as different as insect and horses. This conveys that animals segmented limbs have comparable material characteristics.

For comparative anatomists global models as the spring mass model are less informative since morphometric data normally are not involved in the models. It is possible to introduce them into a model, by distributing the elasticity (of the spring) onto the joints and thus introducing a massless polysegmental kinematic connected with rotational springs 
(Fig 1C). The stiffness is then the ratio variation of the joint torque over variation of the joint aperture i.e. the slope of the tangent at the torque-aperture characteristics.

Seyfarth et al. [53] studied the stability of a massless three segmented leg very much in detail ( for equations see [53] ) in order to describe its stabilizing behaviour in dependence to its initial configuration (zig-zag or arc like) and stiffness laws (constant or variable). The model assumes that the rotational springs act at the joints in a quasi elastic mode without dissipation i.e. joint torques have the form $T_{i}=k_{i}\left(\phi_{-}-\phi_{o}\right)^{v} \quad$ (Eq. 1). That is the torques - angle characteristics do not show hysteresis. Neglecting the torques at the foot pad, the torques equilibrium equation then simplifies into $T_{1} d_{1}+T_{2} d_{2}=0$ (Eq. 2) (figure 1B). Seyfarth et al. assumed a symmetric loading of the limb during stance phase: the compression of a zig-zag like kinematical chain depends on the stiffness of its joints. With similar values of joints stiffness, a load results in a flexion of all joint angles at a time to the same extent, else the segmental chain would collapse first at the joint of less stiffness than others. But studies on the intralimb kinematics of small mammals [27, 24] and birds [1, 58] during running show that changes occur in all joints at the same time and thus point to symmetrical loading and stiffness equilibrium.

Assuming symmetrical loading the torques equilibrium equation (Eq. 2) then simplifies into $\mathrm{k}_{12} / \mathrm{k}_{23}=\mathrm{l}_{1} / \mathrm{l}_{3}$ (figure $1 \mathrm{~B}$ ) whereas the length of the second segment vanish from the equations [47]. Numerical simulations of in place hopping of this elastic limb model lead to the following result: stability is enhanced if a short first segment correspond a long third segment and reciprocally [47]. The figure 1E presents limb geometry of some terrestrial birds. Birds seem to follow this simple "rule".

Actually no quadruped model has been studied in the same detail. One prerequisite is a better experimental knowledge of the torques-aperture characteristics in quadruped mammals in order to limit the parameter space in the simulations. The experimental determination of the torque-aperture relationships (and following of the stiffness laws) at the level of each joint is accessible performing inverse dynamic calculations of the limbs. These experimental characteristics will also point out the level (distal versus proximal) at which energy dissipation occurs in the limbs during a cycle of gallop. Spring-mass models disregard up to now fully energy dissipation up to the theoretical work of Berkemeier [4]. Inverse dynamic analysis is in small quadruped mammals a rather difficult but realizable task [55] since it is necessary to record separately the force exerted by each limbs. Outgoing from the torque-aperture relationships numerical simulations of a segmented biomechanical model of a quadruped with few degrees of freedom could be calculated looking for "optimal" limb proportion with regard to the robustness of stability i.e. the degree of disturbance the 
structure will accept to overcome without falling. The robustness of stability can be considered as an optimization criterion in the frame of the mechanical self-stabilization hypothesis.

\section{Concluding remarks}

The influence of morphology onto stability cannot be understood intuitively and necessitates the use of numerical simulations. Stability emerges for some value of the parameters - segment's length, articular stiffness...- and is fundamentally unpredicable. The hypothesis that intrinsic stability of the musculo-skeletal mechanics exerted an evolutionary pressure that lead to the real body proportions was recently sustained by some simulation studies [52] [33] which found a good correspondance between the values of the parameters that stabilize spring mass models of humans and small mammals running on one hand and the experimental values of stiffness and angle of attack on the other hand. If stability generally results from the common action of the nervous and mechanical system, the nervous system can find in a self-stabilized mechanics a way to delegate and thus to improve one part of its work particularly at high speed where the reaction times have to be kept short. This would accord well with the decentralized structure of neural control (see Viala et al. and Jamont et al. in this issue).

Actual small mammals, which size is comparable to the oldest fossils of mammals, are a common point of interest for the palaeontology and functional morphology. They are good candidates for the required experimental determination of the torques-aperture characteristics. Their body size enables for the most precise localization of all joint positions including the most proximal using monoplanar videoradiography in a two dimensional frame. Moreover their size enables for the measurement of the ground reaction forces over many locomotor cycles, a prerequisite for a valuable integration of the ground reaction forces and thus for the determination of the position of the centre of mass. Thus small mammals are appropriate to perform inverse dynamic calculations combining high speed videoradiography (500 fps), force measurement and complementary electromyography. The synchronization of all these signals is 100 years after Marey's effort to capture the instant in the movement a realizable [29][22] but exceptional task in integrative animal motion studies. Dynamic simulations on "hybrid" models of model B and C (fig.1) should reveal whether the enhancement of the robustness of the mechanical self-stabilisation during locomotion acted as one determinant factor during evolution leading to actual body proportions.

\section{Références}


[1] A. Abourachid, Bipedal locomotion in ratites (Paleognatiform): examples of cursorial birds, Ibis 142 (2000) 538549.

[2] R. McN. Alexander, Elastic Mechanisms in Animal Movement, Cambridge University Press, 1988, Cambridge UK.

[3] R. McN. Alexander, N. J. Dimery, R.F. Ker, Elastic structure in the back and their role in galloping insomes mammals. J. Zool (Lond) A207 (1985), 467-482.

[4] M. Berkemeier. Modelling the dynamics of quadruped running. Int. J. Rob. Res. 17-9 (1998).

[5] N.A. Bernstein, Some emergent problems of the regulation of motor acts. In Human motor actions. Whiting, H.T.A. (Ed.), North-Holland/Elsevier, Amsterdam, 1984, p343-371.

[6] A. Biewener, Locomotory stresses in the limb bones of two small mammals: the ground squirrel and chipmunk. J. Exp. Biol. 103 (1983) 131-154.

[7] R. Blickhan, The spring mass model for running and hopping, J. Biomech. 22 (1988) 1217-1227.

[8] R. Blickhan and R. Full, Similarity in multilegged locomotion: Bouncing like a monopode. J. Comp. Phys. A Sensory, Neural and Behavioral Physiology, 173:5(1993) 509-517.

[9] R. Brook, Artificial Life and Real Robots, Toward a Practice of Autonomous Systems: proceedings of the first European conference on Artificial Life. Francisco J. Varela, Paul Bourgine, editors, MIT Press. 1992

[10] M. Cartmill, P. Lemelin, D. Schmitt, Support polygons and symmetrical gaits in mammals. Zool J Linn Soc 136(2002) 401-420

[11] G.A. Cavagna, Force platforms as ergometers. J. Appl. physiol. 39:1 (1975).

[12] G.A. Cavagna, N.C. Heglund and C.R. Taylor, Mechanical work in terrestrial locomotion: two basic mechanisms for minimizing energy expenditure, Amer. J. Phys. 233(5) (1977) 243-261.

[13] Cheng, R.B. Stein, K. Jovanovic, K. Yoshida, D.J. Bennett, and Y.Han, Identification, Localization, and Modulation of Neural Networks for Walking in the Mudpuppy (Necturus Maculatus) Spinal Cord, J. Neuroscience 18(11)(1998)4295-4304

[14] S. Collins, A. Ruina, R. Tedrake, M. Wisse, Efficient Bipedal Robots Based on Passive Dynamic Walkers, Science Magazine, Vol. 307 (2005) 1082-1085.

[15] S. H. Collins, M. Wisse, and A. Ruina, A three-dimensional passive-dynamic walking robot with two legs and knees, Int. J. Rob. Res. vol. 20, no. 7, pp. 607-15, 2001.

[16] C.T. Farley, J. Glasheen, T.A. Mc Mahon, Running springs: Speed and animal size. J.Exp.Biol. 185 (1993) $71-86$.

[17] C.T. Farley and O. Gonzalez, Leg stiffness and stride frequency in human running. J. Biomech. 29 (2) (1996) 181186.

[18] M.A. Fedak, N.C. Heglund and C.R. Taylor, Energetics and mechanics of terrestrial locomotion. II. Kinetic energy changes of the limbs and body as a function of speed and body size in birds and mammals. J. Exp. Biol. 79 (1982), 2340 .

[19] A.G. Feldman, Once more on the equilibrium point hypothesis ( $\Lambda$ model) for motor control, J. Motor Beh. 18(1) (1986) 17-54.

[20] M.S. Fischer, Crouched posture and high fulcrum, a principle in the locomotion of small mammals. The example of the rock hyrax (Procavia capensis) (Mammalia. Hyracoidea). J. Hum. Evol. 26 (1994) 501 -524.

[21] M.S. Fischer, The locomotion of Procavia Capensis (Mammalia, Hyracoidea): Zur Evolution des Bewegunssystems bei Säugetieren. Abhandlungen des Naturwissenschaftlichen Vereins, Hamburg, 1998, Goecke \&Evers Keltern-Weiler (ed.).

[22] M.S. Fischer, Kinematics, EMG, and Inverse-dynamics of the therian forelimb - a synthetical approach. Zool Anz. 238 (1999), 41-54.

[23] M.S. Fischer and R. Lehmann, Application of cineradiography for the metric and kinematic study of in-phase gaits during locomotion of the pika (Ochotona rufescens, Mammalia: Lagomorpha). Zoology 101 (1998) 148-178.

[24] M.S. Fischer, N. Schilling, M. Schmidt, D. Haarhaus and H. Witte, Basic limb kinematics of small therians mammals. J. Exp. Biol. 205(9) (2002) 1315-1338.

[25] R.J. Full and Koditschek, Templates and anchors: neuromechanical hypotheses of legged locomotion on land, J. Exp. Biol. 202 (1999) 3325-3332.

[26] M. Garcia, A. Ruina, A. Chatterjee and M. Coleman, the simplest walking model: stability, complexity and scaling. ASME J. Biomech. Eng. 120(2) (1998) 281-288.

[27] J.P. Gasc, Asymmetrical gait of the Saharian rodent Meriones shawi shawi (Duvernoy, 1842) (Rodentia, Mammalia): a high speed cineradiographic analysis. Can. J. Zool. 71 (1992) 90-98.

[28] J.P. Gasc, Comparative aspects of gait, scaling and mechanics in mammals. Comp. Bioch. Phys. Part A 131 (2002) $121-133$.

[29] J.P. Gasc, D. Cattaert, C. Chasserat, F. Clarac, Propulsive Action of a snake pushing against a single site: its combined analysis, J. Morph 201 (1989) 315-329.

[30] S. Grillner, P. Wallen, L. Brodin, Neural Network generating locomotor behaviour in lamprey: circuitry, transmitters, membranes properties and simulation, Annu. rev. Neurosci. 14 (1991) 169-199. 
[31] T.Mc Geer, Passive walking with knees, Proceedings of the IEEE International Conference on robotics and Automation, Cincinnati, OH (1990) 1640-1645.

[32] A. Goswami, B. Thuilot, and B. Espiau, A study of the passive gait of a compass-like biped robot: Symmetry and chaos, Int. J. Rob. Res., vol. 17, no. 12, pp. 1282-301, 1998.

[33] R. Hackert, H. Witte, Spinal flexion contributes to self-stabilization of pikas' half-bound. Arch. Phys. Bioch. 111 (Supp.)(2002)

[34] R. Hackert, H. Witte H, M.S. Fischer, Dynamics of half-bound and spinal flexion.- In: R. Blickhan, R. (Hrsg.), Motion Systems 2001. Collected short papers of the Innovationkolleg "Bewegungssysteme", Shaker Verlag, Aachen,2001, p137 - 141.

[35] R. Hackert and M.S. Fischer, Aspect of spinal bending in small mammals, submitted to J Exp Biol.

[36] K. Hase, N. Yamazaki, Computational Evolution of human bipedal walking by a neuro muscular skeletal model. Proceedings of third Symp. on artificial life and robotics, iota japan, (1998) 174-177.

[37] N.C. Heglund, G.A. Cavagna and C.R. Taylor (1982). Energetics and mechanics of terrestrial locomotion. III. Energy changes of the center of mass as a function of speed and body size in birds and mammals, J. Exp. Biol. 79, 4156.

[38] N.C. Heglund, C.R. Taylor, Speed, stride frequency and energy cost per stride: how do they change with body size and gait, J. Exp. Biol. 138 (1982).

[39] A. D. Kuo, "Stabilization of lateral motion in passive dynamic walking”, Int. J. Rob. Res., 18(9), pp. 917-30, 1999.

[40] A.N. Kuznetsov, Energetical profit of the third segment in parasagittal legs, J. theor. Biol. 172 (1995) 95-105

[41] S. Labeit, B. Kolmerer, Titin: giant proteins in charge of muscle ultrastructure and elasticity, Science 270 (1995) 293-296.

[42] K. Lilje, C. Tardieu, M.S. Fischer, Scaling of long bones in ruminants with respect to the scapula, Journal of zoological systematics and evolutionary research, 41 (2)(2003) 118-126.

[43] W.A Linke, Stretching molecular springs: elasticity of titin filaments in vertebrate striated muscles. Histo Histopatolog. 15 (2000) 799-811

[44] E.-J. Marey, Analyse des mouvements du cheval par la chronophotographie, La Nature 1306 (1898) 22-26.

[45] T.A. Mc Mahon, Role of compliance in mammalian running gaits, J. Exp.Biol. 115 (1985) 263-282.

[46] T.A. Mc Mahon and G.C. Cheng, The mechanics of running: How does stiffness coupled with speed? J. Biomech. 23 (Suppl 1.)(1990) 65-78.

[47] B. Scheppens, P.A. Willems, and G.A.Cavagna, The mechanics of running in children, J. Physiol. 509(3) (1998) 927-940.

[48] N. Schilling, and M.S. Fischer, Kinematic analysis of treadmill locomotion of the tree shrews Tupaia glis (Scandentia: Tupaiidae). Zeitschrift für Säugetierkunde 64 (1999) 129-153.

[49] N. Schilling, R. Hackert, M.S. Fischer, Intervertebral movements during locomotion in small mammals, J Morph. 260(3)(2004).

[50] M. Schmidt and M.S. Fischer, Cineradiographic study of forelimb movements during quadrupedal walking in the brown lemur (Eulemur fulvus, Primates: Lemuridae). Amer. Journal of phys. Anthrop. 111 (2000) 245-262.

[51] W.J. Schwind, Spring loaded inverted pendulum running: A plant model. Dissertation University of Michigan, USA, 1998.

[52] A. Seyfarth, H. Geyer, R. Blickhan. A movement criterium for running. J. Biomech. 35 (2002) 649-655.

[53] A. Seyfarth, M. Günther, R. Blickhan, Stable operation of an elastic three segmented leg. Biol. Cybern. 84 (2001) 365-382.

[54] A. Seyfarth, H. Geyer, H Herr, Swing-leg retraction: a simple control model for stable running, J. Exp. Biol. 206 (2003) 2547-2555.

[55] G. Taga, Self-organized control of bipedal locomotion, Biol Cybern. 65 (1991) 147-159.

[56] A.J. Ijspeert, A connectionist central pattern generator for the aquatic and terrestrial gaits of a simulated salamander, Biol. Cybern. 84(5)(2001) 331-348.

[57] L. Tskhovrebova, J.A. Trinick, J.A. Sleep and R.M. Simmons, Elasticity and unfolding of single molecules of the giant muscle protein titin. Nature 387 (1997) 308.

[58] M Verstappen, P Aerts and R Van Damme, Terrestrial locomotion in the black-billed magpie: kinematic analysis of walking, running and out-of-phase hopping J. Exp. Biol., 203(14) 2000, 2159-2170.

[59] H. Wagner, R. Blickhan, Stabilizing function of skeletals muscles: an analytical investigation. J. Theor. Biol 199 (1999) 163-179.

[60] H. Witte, J. Biltzinger, R. Hackert, C. Reich, N. Schilling, M.S. Fischer, Torques patterns of the limbs of small therian mammals during locomotion on flat ground. J. Exp. Biol. 205(9)(2002), 1339-1353. 

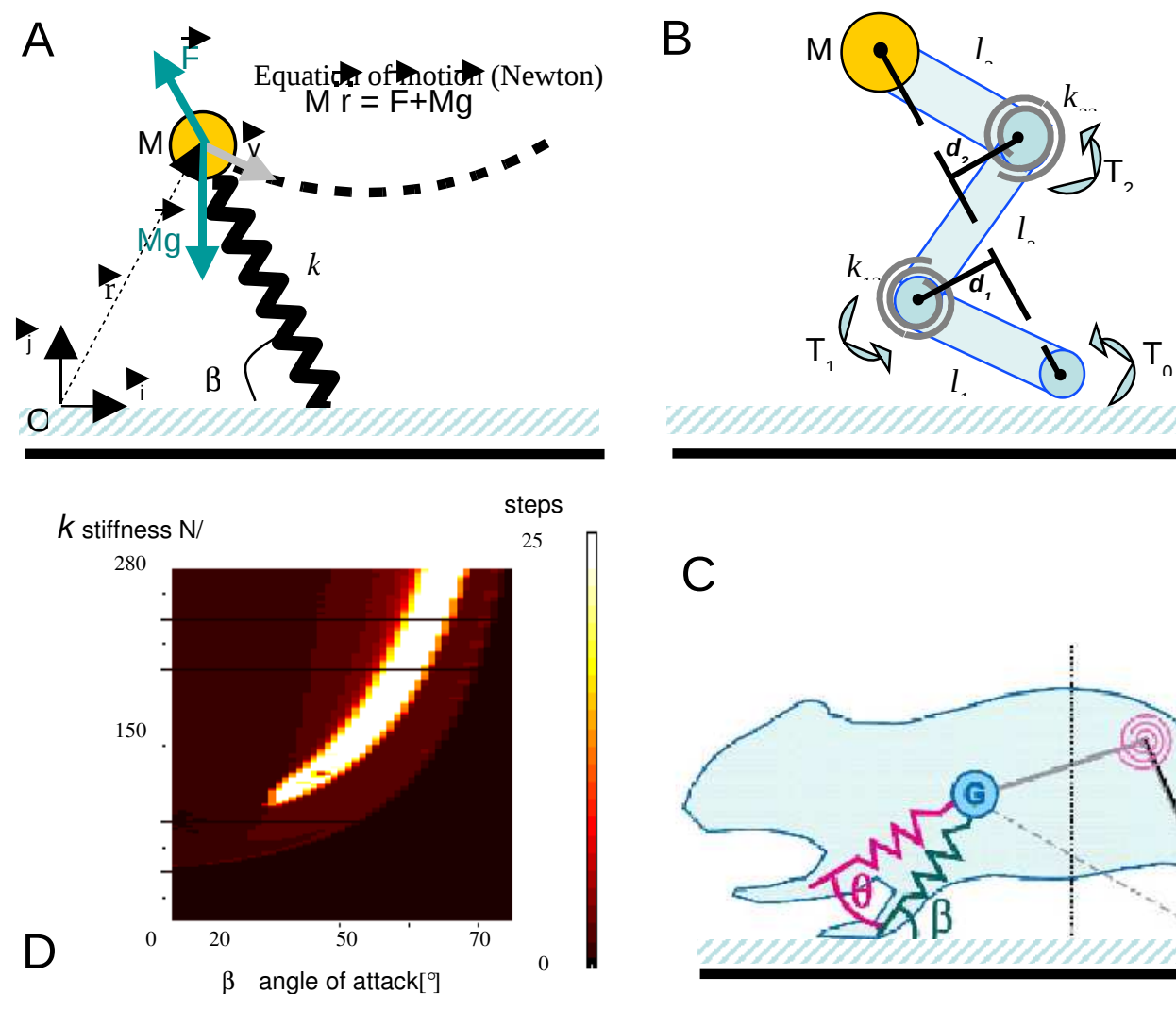

C

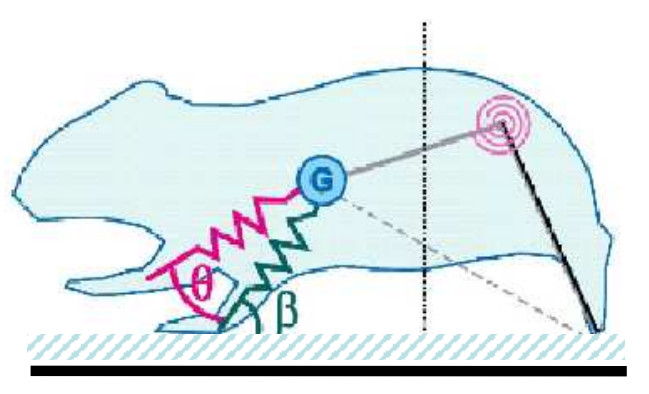

王

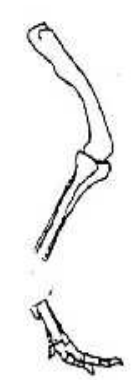

Al
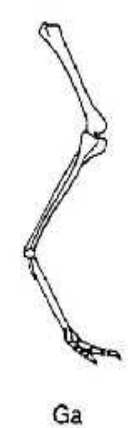
Allosaurus

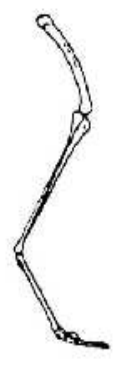

\$n Sinornithoides

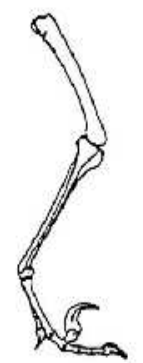

De

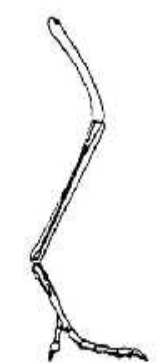

(A)

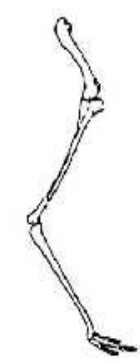

(A)

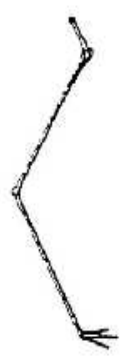

(F)

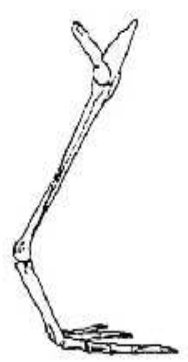

(1.)

Deinonychus Archaeopteryx Rhea Fiamingo

Hesperornis

\section{Caption of figure 1}

A) The spring mass system for running and hopping in humans (Blickhan [7]): the position vector $\mathbf{r}$ can be computed integrating numerically the equation of motion. Model parameters are speed vector $\mathbf{v}$, mass $\mathbf{M}$, stiffness $k$, length of the 
spring and angle of attack $\beta$ at touch down. B) Distribution of the spring elasticity onto rotational springs located at the articulations. In this way morphological parameters as segment length are introduced into the models. (Seyfarth [53]) C) Spring mass model of a half-bounding small mammal (Hackert [33]). Back flexion/extension translates the centre of mass horizontally during half-bound leading to adjustments of the angle of attack $\beta$. D) The dynamic stability of the spring mass system. The simulations were done for each combination of the stiffness and the angle of attack. The colours represent the number of steps up to 25 performed stably by the model. An extended J-region of stability exists for $\beta$ larger than a threshold value of about $25^{\circ}$. E) Limbs of some terrestrial birds: A result of the numerical simulation of the dynamics of the three segmented spring limb with symmetrical loading: A short femur correspond a long third segment and vice versa. Does dynamic stability constraint the evolution of our morphology?

Figure 1: A) Le système masse ressort pour la course et le saut (Blickhan [7]): le vecteur position $\mathbf{r}$ est calculé en intégrant numériquement l'équation vectorielle du mouvement. Les paramètres du modèle sont le vecteur vitesse v, la masse $\mathrm{M}$, la raideur $k$, la longueur à vide du ressort et l'angle d'attaque $\beta$ à l'instant du posé. B) Distribution de l'élasticité du ressort sur des ressorts de torsion associé aux articulations. Ainsi des paramètres morphologique tells la longueurs des segments peut être introduite dans le modèle. (Seyfarth [53]) C) Modèle masse ressort d'un petit mammifère pratiquant le half-bound (Hackert [33]). L'extension/flexion du dos translate le centre de masse horizontalement pendant le half-bound conduisant à des ajustements de l'angle d'attaque $\beta$. D) La stabilité dynamique du système masse-ressort. La dynamique a été simulée pour chaque combinaison de la raideur et de l'angle d'attaque. Les couleurs représentent le nombre de cycles locomoteurs réalisés stablement par le modèle. Une région de stabilité étendue en forme de $\mathbf{J}$ existe pour des valeurs de $\beta$ plus grande qu'une valeur seuil ici $25^{\circ}$. E) Les membres de quelques oiseaux terrestres: Un résultat de la simulation numérique de la dynamique d'une jambes élastique trisegmentée avec chargement symétrique : A un court premier segment correspond un troisième long segment et vice versa. La stabilité dynamique contraint-elle l'évolution de notre morphologie? 